\title{
A nonlinear model of the hand-arm system and parameters identification using vibration transmissibility
}

\author{
Nahid Hida, Mohamed Abid, and Faouzi Lakrad ${ }^{\mathrm{a}}$ \\ Laboratory of Renewable Energy and Dynamics of Systems, Faculty of Sciences Aïn Chock, \\ University Hassan II-Casablanca, Casablanca, Morocco
}

\begin{abstract}
In the present paper a lumped single degree-of-freedom nonlinear model is used to study biodynamic responses of the hand arm system (HAS) under harmonic vibrations. Then, the harmonic balance method is implemented to derive the vibration transmissibility. Furthermore, Padé approximations are used in the identification process of biodynamic characteristics of the HAS model. This process is based on minimizing the distance between the theoretical and the experimentally measured transmissibilities.

The proposed identification workflow is applied to vibrations at the wrist in two cases: 1) the transmissibility versus the grip force for fixed excitation frequencies, and 2) the transmissibility versus the excitation frequency for fixed grip force.
\end{abstract}

\section{Introduction}

Lumped parameters linear models are widely used in the literature to study the biodynamic responses of the human hand-arm system (HAS) to vibration, see for instance [1] and [2]. These models range from single to multiple degrees of freedom mass-spring-damper systems. The corresponding coefficients are identified using fitting and optimization techniques that minimize the distance with measured mechanical impedances and/or vibration transmissibilities to different segments of the HAS.

In fact, these models impose constant stiffness, mass and damping coefficients while the skin-muscle-bone characteristics change with changing experimental conditions and their mechanical behaviors are knowledged to be nonlinear. Indeed, many factors highly influence the HAS vibration response, we cite among others, frequencies and amplitudes of the imposed vibrations, hand forces (the grip and the push-pull forces of the hand on the handle), the posture, the vibration direction, the handle geometry and the subject anthropometry, see [3] and [4].

Many contradictory results are reported in the literature because of the differences in the experimental conditions [3]. However, all experimental studies agreed that the transmissibility of the hand-arm segments show rapid decrease with increasing frequency and distance from the source of vibration [3]. Moreover, it is found that vibrations above 200 $\mathrm{Hz}$ are confined to the hand, while frequencies below 100 $\mathrm{Hz}$ and $40 \mathrm{~Hz}$ could be transmitted to the forearm and the upper arm, respectively.

In the present work, a nonlinear lumped single degree-offreedom system is used to model the HAS. Then, the vibration transmissibility at the wrist is used to identify the parameters of this model under various conditions of the hand forces and the excitation frequencies. The main parameters of control of the HAS model are taken nonconstant and are expressed as fractional polynomials of the grip force or the

\footnotetext{
a e-mail: f.lakrad@fsac.ac.ma
}

excitation frequency.

The present paper is organized as follows: in section 2, a nonlinear mathematical model of the HAS is presented and an expression of the vibration transmissibility is derived. In section 3, the process of parameters identification is explained and applied to two case studies.

\section{Mathematical model}

A mathematical single degree-of-freedom model of nonlinear vibrations of the HAS is shown in Fig.1. The viscoelastic characteristics of the HAS are modelled by restoring and dissipative forces that are assumed to be nonlinearly related to the displacement and the velocity, respectively. In what follows, cubic polynomial nonlinearities are considered.

The absolute displacement of the mass is denoted $x(t)$ and

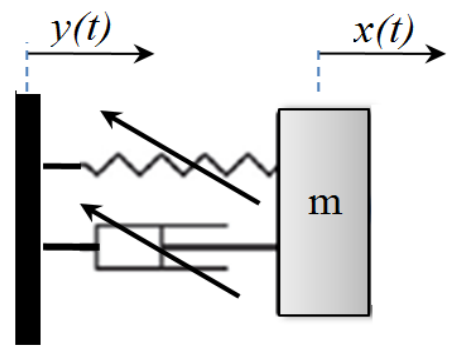

Fig. 1. The studied non-linear lumped hand-arm model. $y(t)$ is the imposed displacement at the handle.

the imposed harmonic displacement at the handle is denoted $y(t)=Y \cos (\Omega t)$, where $t$ is time, $Y$ and $\Omega$ are the amplitude and the frequency (measured in $\mathrm{rad} / \mathrm{s}$ ), respectively.

The nonlinear equation of motion of the mass is written as 
follows

$$
\begin{array}{r}
m \ddot{x}+c_{1}(\dot{x}-\dot{y})+k_{1}(x-y)+c_{3}(\dot{x}-\dot{y})^{3} \\
+k_{3}(x-y)^{3}=0
\end{array}
$$

where $k_{1}$ and $c_{1}$ are the linear stiffness and viscous damping coefficients, respectively. The corresponding nonlinear coefficients are denoted $k_{3}$ and $c_{3}$.

A first order approximation of the periodic solution of Eq.(1), having the same frequency $\Omega$ as the imposed harmonic displacement of the handle, is obtained using the harmonic balance method (HBM) [5].

Thus, the solution is sought under the following form

$$
x(t)=X \cos (\Omega t-\theta)
$$

where $X$ and $\theta$ are the amplitude and the phase of the absolute displacement. In Eq.(2) the contribution of higher order harmonics are assumed negligible.

The amplitude $X$ is given by

$$
X=\sqrt{Y^{2}+a^{2}+\frac{a^{2}\left(3 k_{3} a^{2}+4 k_{1}-4 m \Omega^{2}\right)}{2 m \Omega^{2}}}
$$

where $a$ is the the relative displacement amplitude of the mass. It is solution of the following sixth order algebraic equation

$$
\begin{array}{r}
\frac{9}{16}\left(\Omega^{6} c_{3}^{2}+k_{3}^{2}\right) a^{6}+\frac{3}{2}\left(k_{3}\left(k_{1}-m \Omega^{2}\right)+c_{1} c_{3} \Omega^{4}\right) a^{4} \\
+\left(\left(k_{1}-m \Omega^{2}\right)^{2}+c_{1}^{2} \Omega^{2}\right) a^{2}=m^{2} Y^{2} \Omega^{4}
\end{array}
$$

It is worth noting that nonlinearities i.e., $k_{3}$ and $c_{3}$ are causing the existence of polynomial terms of order 4 and 6 in Eq. (4) and are leading to the possibility of coexistence of three periodic solutions.

The transmissibility of the displacement is given by

$$
T R=\frac{X}{Y}=\sqrt{1+\frac{a^{2}}{Y^{2}}\left(\frac{3 k_{3} a^{2}+4 k_{1}-2 m \Omega^{2}}{2 m \Omega^{2}}\right)}
$$

Combining Eqs. (4) and (5), the transmissibility can be expressed as

$$
T R=\frac{a}{m Y \Omega^{2}} \sqrt{\left(\frac{3}{4} k_{3} a^{2}+k_{1}\right)^{2}+\Omega^{2}\left(\frac{3}{4} \Omega^{2} c_{3} a^{2}+c_{1}\right)^{2}}
$$

In order to use the derived nonlinear transmissibility given in Eqs. (5) or (6), the amplitude $a$ of the relative displacement should be measured. Otherwise, it becomes an unknown of the optimization problem and physically meaningful constraints should be imposed to identify it.

The linear case is obtained by cancelling the effects of nonlinearities i.e., setting $k_{3}=c_{3}=0$. Hence, the amplitude of the linear relative displacement is

$$
a_{L}=\frac{m Y \Omega^{2}}{\sqrt{\left(k_{1}-m \Omega^{2}\right)^{2}+c_{1}^{2} \Omega^{2}}}
$$

Consequently, the classical linear transmissibility $\mathrm{TR}_{L}$ is given by

$$
T R_{L}=\sqrt{\frac{k_{1}^{2}+c_{1}^{2} \Omega^{2}}{\left(k_{1}-m \Omega^{2}\right)^{2}+c_{1}^{2} \Omega^{2}}}
$$

It is worth pointing out that the linear transmissibility (8) is independent of the imposed handle motion amplitude $Y$, while the nonlinear transmissibility (6) is dependent on it. Based on vibration measurements on the HAS, Adewusi et al. [3] found experimentally, using two different magnitudes of broad-band random vibrations of the handle, that the excitation magnitude affects the transmissibility, particularly around the characteristic frequencies.

\section{Parameters identification}

The model parameters identification process is based on experimental measurements of the HAS vibration transmissibility, along the forearm direction i.e., $Z_{h}$ axis, that were performed on nine subjects under various hand forces and excitation frequencies [4].

Experiments were conducted for a hand-arm posture corresponding to bent-arm with $90^{\circ}$ elbow angle. Pan et al. [4] used a six-degree polynomial to fit the experimental transmissibility in terms of the grip force. Then, they computed the mean transmissibility of the nine subjects.

In the present paper, two case studies of the wrist vibration transmissibility are investigated. For each case the transmissibility is measured while varying a parameter of control. Then, in each case a parameter of the model is chosen to be expressed in terms of the parameter of control.

In the first case study, the control parameter that is varied is the grip force $F_{g}$. Specifically, four discrete sinusoidal vibrations with frequencies $10 \mathrm{~Hz}, 16 \mathrm{~Hz}, 25 \mathrm{~Hz}$ and $40 \mathrm{~Hz}$ are used as imposed displacement on a cylindrical handle with a constant velocity of $0.1 \mathrm{~m} / \mathrm{s}$. The corresponding imposed accelerations on the handle-arm interface are, respectively, $6.28 \mathrm{~m} / \mathrm{s}^{2}, 10.05 \mathrm{~m} / \mathrm{s}^{2}, 15.71 \mathrm{~m} / \mathrm{s}^{2}$ and $25.13 \mathrm{~m} / \mathrm{s}^{2}$.

In this first case study, the linear transmissibility model (8) is used and the linear stiffness $k_{1}$ is expressed in terms of the grip force $F_{g}$ using the following Padé approximation

$$
k_{1}=\frac{\alpha_{0}+\alpha_{1} F_{g}+\alpha_{2} F_{g}^{2}+\alpha_{3} F_{g}^{3}}{\beta_{0}+\beta_{1} F_{g}+\beta_{2} F_{g}^{2}}
$$

where $\alpha_{i}, i=0 . .3$ and $\beta_{j}, j=0 . .2$ are constants to be identified.

In the second case study, the transmissibility is measured while varying the control parameter that is the excitation frequency, for fixed values of hand forces. The nonlinear transmissibility model (6) is used in the process of identification. The relative displacement amplitude $a$ is expressed in terms of the excitation frequency $\Omega$ using the following fractional polynomials form

$$
a=\frac{a_{0}+a_{1} \Omega+a_{2} \Omega^{2}+a_{3} \Omega^{3}}{a_{4}+a_{5} \Omega+a_{6} \Omega^{2}}
$$

In both cases the model parameters are identified by minimizing the distance between the theoretical formulas of the transmissibility (8) or (6) and the measured mean transmissibility at the wrist [4].

Furthermore, since the results of the minimization problem are not unique and not surely global, additional physically meaningful constraints are to be imposed to the parameters of the model. Hence, the following constraints are imposed on the linear parameters

$0<m \leq 3[\mathrm{Kg}] ; 50<c_{1}<500[\mathrm{~N} . \mathrm{s} / \mathrm{m}] ; k_{1}>0[\mathrm{~N} / \mathrm{m}]$ 
The imposed constraint on the relative displacement amplitude $a$, used in the second case study, is

$$
0 \leq a \leq Y(1+T R)
$$

In the present work, the nonlinear stiffness and damping coefficients are not explicitly expressed in terms of the control parameters.

\subsection{Application of the linear model}

The nine parameters of the linear model to be identified are the mass $m$, the linear damping coefficient $c_{1}$ and the seven parameters, $\alpha_{i}$ and $\beta_{i}$, defining the linear stiffness $k_{1}$ given in Eq. (9).

The outcome of the optimization process, in the case of $10 \mathrm{~Hz}$ excitation, is that the identified mass and damping coefficient are $m=2.45 \mathrm{Kg}$ and $c_{1}=104.34 \mathrm{~N} . \mathrm{s} / \mathrm{m}$. The expression of the linear stiffness in terms of the grip force is given by

$k_{1}=\frac{0.175053-0.024855 F_{g}-0.005343 F_{g}^{2}+9.0 \times 10^{-6} F_{g}^{3}}{0.000016-0.000008 F_{g}-1.49 \times 10^{-7} F_{g}^{2}}$

The corresponding sum of squares error (SSE), between the theoretical and the measured transmissibilities, is equal to 0.0016 . This low value of SSE proves the high accuracy of the theoretical model.

Figure 2 shows a good agreement between the computed linear transmissibility (represented in lines) and the experimental transmissibilities (in symbols) versus the grip force for four excitation frequencies (in different colors). Figure 2 shows that increasing the excitation frequency leads to decreasing the transmissibility at the wrist. Moreover, for each frequency the transmissibility increases with increasing the grip force till reaching a saturation-like phenomenon or starting to decrease. The increasing transmissibility phase is longer with increasing the excitation frequency.

In Fig. 3 are shown the predicted natural frequency $\omega$ and

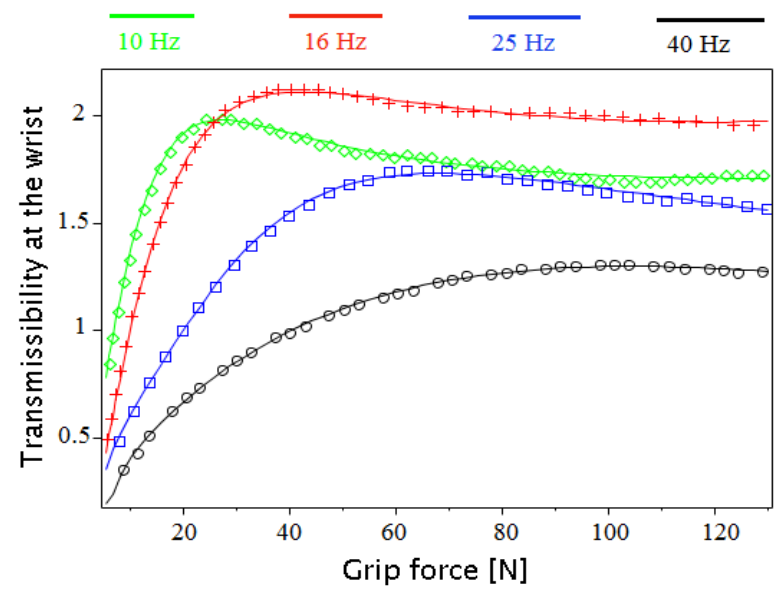

Fig. 2. The transmissibility at the wrist versus the grip force for various excitation frequencies. The lines correspond to the theoretically computed transmissibility. The measured data [4] are shown in symbols $(\diamond+\square, 0)$. the damping ratio $\xi$ versus the grip force for $10 \mathrm{~Hz}$ sinusoidal frequency excitation. They are defined as follows

$$
\omega=\sqrt{\frac{k_{1}}{m}} \quad \text { and } \quad \xi=\frac{c_{1}}{2 m \omega}
$$

Figure 3 shows that the natural frequency is increasing and the damping ratio is decreasing with increasing the grip force. In fact, increasing the grip force rigidifies the system and consequently increases the natural frequency. Moreover, the increase rate of the natural frequency is decreasing with increasing $F_{g}$.
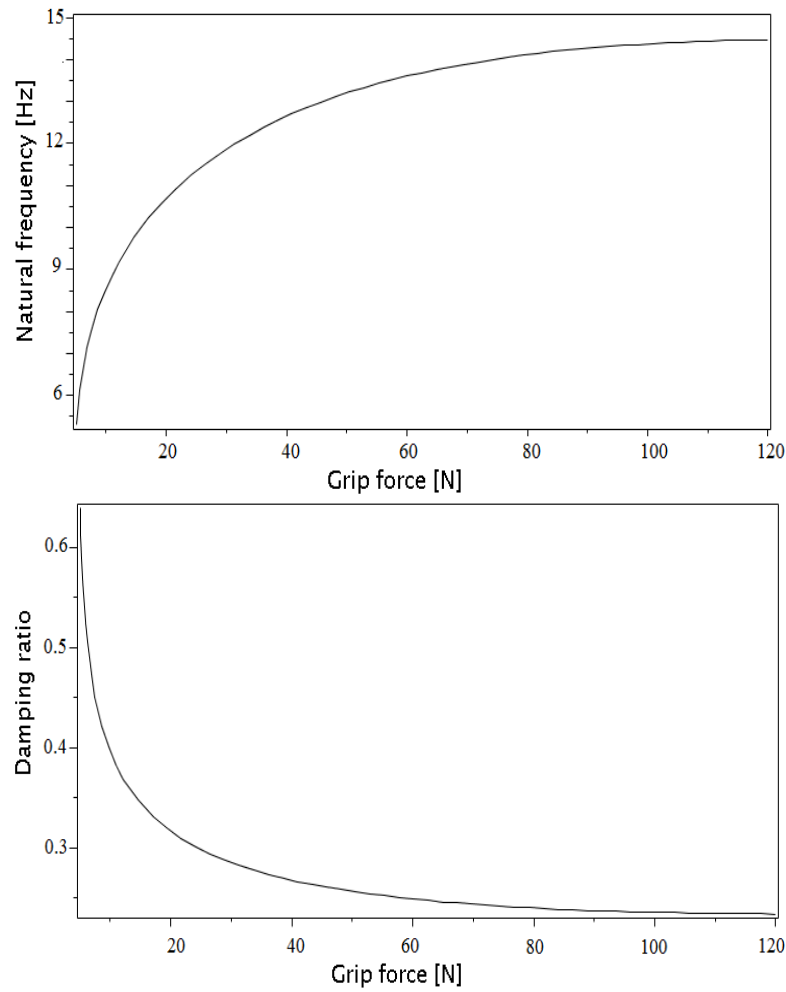

Fig. 3. Variation of the natural frequency and the damping ratio versus the grip force for the excitation frequency $10 \mathrm{~Hz}$.

\subsection{Application of the nonlinear model}

In this subsection, the developed nonlinear transmissibility (6) is studied versus the excitation frequency for a fixed grip force and for varying amplitude $Y$. Indeed, the linear transmissibility (8) is independent of the handle displacement amplitude $Y$. Consequently, changing $Y$ will not affect the curve of the transmissibility versus any parameter of control.

Experimentally, the transmissibility was measured, at various locations of the HAS, versus the excitation frequency for various values of the hand forces in [4]. The imposed velocity amplitude $V$ at the handle was taken constant equal to $0.012 \mathrm{~m} / \mathrm{s}$, and the corresponding imposed displacement amplitude is $Y=V / \Omega$.

Since the relative displacement amplitude $a$ was not measured in [4], then six parameters of the nonlinear model are to be identified : the amplitude $a$, the mass, the linear and nonlinear stiffness and damping coefficients. 
Furthermore, in order to perform the optimization process, the amplitude $a$ is expressed as a fractional polynomial of the excitation frequency $\Omega$, see Eq.(10).

Figure 4 shows the good fit between the results of the nonlinear transmissibility given in Eq. (6) and the experimentally measured transmissibility at the wrist for $75 \mathrm{~N}$ grip force [4].

Figure 4 shows a resonant frequency of the wrist, between $12 \mathrm{~Hz}$ and $17 \mathrm{~Hz}$, that corresponds to the maximum of vibration transmissibility. Moreover, the transmissibility is amplified, i.e., higher than one, for excitation frequencies below $60 \mathrm{~Hz}$. Consequently, low frequencies vibrations are a potential danger for the wrist.

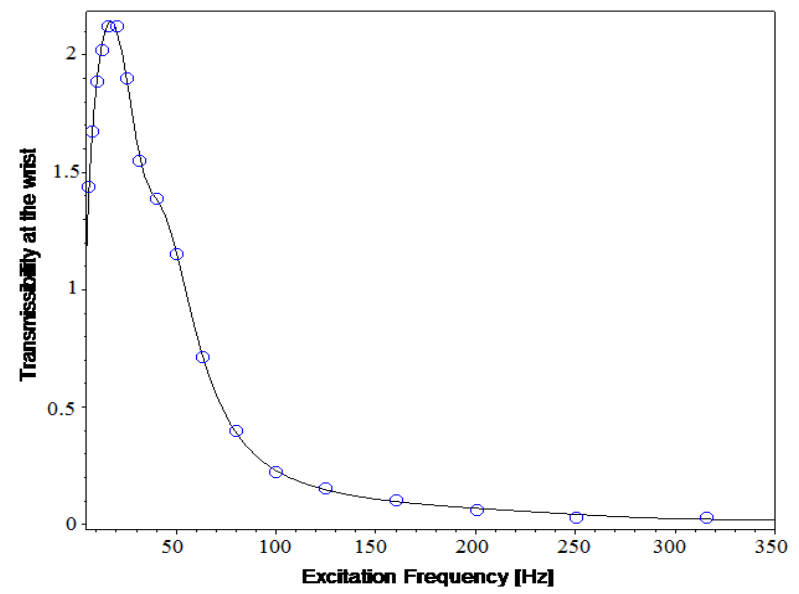

Fig. 4. Transmissibility at the wrist versus the excitation frequency for $75 \mathrm{~N}$ grip force. Experimental data [4] are shown in circles and the theoretical results in continuous line.

\section{Conclusion}

In the present study a nonlinear lumped single degree-offreedom system is used to model the hand arm system response under sinusoidal imposed displacement of the handle. Then, the vibration transmissibility is derived analytically using the harmonic balance method.

Furthermore, the process of the model parameters identification is based on the minimization of the distance between measured and theoretical transmissibilities.

The derived workflow is applied to two case studies: 1) the transmissibility is measured versus the grip force; and 2) the transmissibility is measured versus the excitation frequency.

In order to perform the identification process the linear stiffness is expressed in terms of the grip force in the first case. In the second case, the amplitude of the relative displacement of the mass is taken dependent on the frequency. In both cases, the Padé approximations are used.

The outcome of the present work is that varying parameters models are fitting better to experimental measurements than the constant parameters models. Furthermore, the nonlinear model is introduced and it fits the case where the magnitude of the imposed displacement is varied.

As a continuation of the present work the mass, the stiffness and the damping coefficients will be allowed to vary in terms of influencing factors on the vibration transmissibility. Moreover, the proposed identification process will be extended to higher d.o.f. models.

\section{References}

1. Rakheja S, Wu JZ, Dong RG, Schopper AW. A comparison of biodynamic models of the human hand-arm system for applications to handheld power tools. Journal of Sound and Vibration 249 : 55-82, 2002.

2. Dong RG, Welcome DE, Xu X, Chen Q, Lin H, McDowell TW, Wu JZ. A model for simulating vibration responses of grinding machineworkpiece- hand-arm systems. Journal of Sound and Vibration 431 : 276-294, 2018.

3. Adewusi SA, Rakheja S, Marcotte P, Boutin J. Vibration transmissibility characteristics of the human handarm system under different postures, hand forces and excitation levels. Journal of Sound and Vibration 329 : 2953-2971, 2010.

4. Pan D, Xu XS, Welcome DE, McDowell TW, Warren $\mathrm{C}$, $\mathrm{Wu} \mathrm{J}$, Dong $\mathrm{RG}$. The relationships between hand coupling force and vibration biodynamic responses of the handarm system. Ergonomics, DOI: 10.1080/00140139.2017.1398843, 2017.

5. Nayfeh AH. Perturbation methods. Wiley-VCH Verlag; 2004. 